Longan Phillips, S. (2022). CONTAR LA HABANA VIEJA PRERREVOLUCIONARIA DESDE LA CIUDAD, EL COMERCIO Y TRES HITOS PUNTUALES: DIAMANTES, EL NATIONAL CITY BANK Y LA LLEGADA DE LA REVOLUCIÓN. Revista Herencia, 35(1), enero-junio, 58-69.

\title{
CONTAR LA HABANA VIEJA PRERREVOLUCIONARIA DESDE LA CIUDAD, EL COMERCIO Y TRES HITOS PUNTUALES: DIAMANTES, EL NATIONAL CITY BANK Y LA LLEGADA DE LA REVOLUCIÓN
}

\author{
Telling the Prerrevolucionary Vieja Habana from City, Commerce, and Three Punctual Landmarks: \\ Diamonds, the National City Bank, and the Revolution's Arrival \\ Shirley Longan Phillips \\ Universidad de Costa Rica, Costa Rica \\ shirley.longan@ucr.ac.cr
}

Recibido: $10-10-2021$

Aprobado: 15-11-2021

Shirley Longan Phillips, Universidad de Costa Rica. Máster en Literatura Latinoamericana; Bachiller en Filología Española y Bachiller en inglés. Trabaja en la Escuela de Estudios Generales en la Sección de Comunicación y Lenguaje.

\section{RESUMEN}

La historia de La Vieja Habana prerrevolucionaria ofrece todo un reto para su estudio, tanto hacerlo en el país de Cuba como hacerlo a la distancia. Con ese reto en mente, Ana Vera Estrada entrevistó, durante los últimos meses del año 1999 y los primeros del 2000, a una serie de adultos mayores, para que, desde el recuerdo y la memoria, dibujaran ese espacio llamado La Vieja Habana: sus límites espaciales, reales o simbólicos, el comercio vivo de la ciudad y elementos puntuales que marcaron la vida antes de la Revolución Cubana en 1959, por ejemplo, la industria de diamantes, el robo al National City Bank y la llegada de la Revolución. El presente artículo retoma el texto de Estrada y hace una relación intertextual, para ampliar, a partir de historiografía y bibliografía escrita, los ejes escogidos de la historia oral recolectada por Estrada.

Palabras clave: oralidad; historiografía; relatos orales; ciudad; comercio

ABSTRACT

Studying the History of La Vieja Habana prior to the Cuban Revolution is a challenge, not only in the country itself, but studying abroad. Ana Vera Estrada took this challenge and interviewed some elderly people, during the last months of 1999 and the first months of the year 2000. They draw, from their memories, the space called La Vieja Habana: its spatial and symbolic limits, the vivid commerce downtown, and specific moments of History that changed life before the Cuban Revolution in 1959: the diamonds industry and the National City Bank's assault. This article takes as a basis Estrada's text and makes an intertextual relation, to enhance by historiographic and bibliographical texts, the axes chosen from the oral stories recollected by Estrada.

Keywords: Orality; historiography; oral stories; downtown; commerce 
Revista Herencia, Vol. 35 (1), enero-junio, 2022.

\section{Introducción}

Leer, escribir, contar y estudiar La Habana Vieja prerrevolucionaria supone un gran reto, pues indudablemente, después del denominado triunfo/traición de la revolución (depende de quién lo cuente) toda la dinámica social cubana, y si se quiere latinoamericana, cambió completamente. Sergio Guerra y Alejo Maldonado (2009), en el libro Historia de la Revolución Cubana explican la importancia de este hito:

Sin duda, la Revolución cubana abrió también una nueva etapa en la historia de América Latina, que se ha caracterizado por el desarrollo del movimiento de liberación nacional y los desafíos a la hegemonía de Estados Unidos y la globalización neoliberal. A partir de 1959, se inició una época de gran efervescencia social y política en todo el continente, dominada por significativos combates revolucionarios y antiimperialistas, poderosas luchas obreras, el despertar de importantes sectores campesinos e indígenas, la elevación del espíritu combativo de las masas marginales y las amplias movilizaciones estudiantiles (2009, p. 11).

Sin embargo, como dice Graciela de Garay en el Diccionario de Estudios Culturales Latinoamericanos (2009): "Toda historia [sic] antes de ser escrita fue contada"; por eso, ¿cómo se vivía en La Vieja Habana antes de la revolución? Justamente, con esta pregunta en mente, Ana Vera Estrada entrevistó, durante los últimos meses del año 1999 y los primeros del 2000, a una veintena de adultos mayores para que le dibujaran momentos, lugares, opiniones y acontecimientos de lo que se conoce como La Habana Vieja. Estos testimonios e historias de vida están recogidos en el artículo "Historia contada de la vieja Habana o La Habana relatada” publicado en el libro La oralidad: ¿ciencia o sabiduría popular? compilado por la misma autora y publicado en La Habana en el año 2004 por la Cátedra de Oralidad Carolina Poncet, del Centro de Investigación y Desarrollo de la Cultura Cubana Juan Marinello. El propósito del artículo de la señora Estrada era tender una mirada, a partir de la memoria, de los años previos a la revolución cubana.

El presente artículo toma como base los comentarios de los informantes y amplía, a partir de otros textos sobre la historia cubana, ejes puntuales: los límites de La Vieja Habana, ¿hasta dónde llega la ciudad? Esto permite ilustrar la estructura demográfica y la constitución de los barrios. El segundo eje es el comercio: los extranjeros, los informales, y las divisiones de trabajo en hombres, mujeres y negros. Y, por último, un breve repaso de la Historia con tres hitos más: la talla de diamantes, el robo del Tesoro de la República en el National City Bank y, muy brevemente, la llegada de la revolución en La Habana Vieja. El objetivo es corroborar, y en otros casos precisar, a partir de textos de Historia, lo que los informantes mencionan a partir de su memoria.

\section{¿Desde dónde contar?}

Según el Diccionario de Estudios Culturales Latinoamericanos (2009), en la entrada sobre oralidad escrita por la Graciela de Garay, se explica que entre las formas de comunicación oral se encuentran el testimonio, el cual registra las declaraciones verbales de un testigo ocular de un acontecimiento; también existe la tradición oral o testimonio indirecto, que se pasa de generación en generación, y depende, para su transmisión, de la memoria colectiva. Además, existe la historia oral, o narrativa conversacional, que busca, por medio de entrevistas, saber las experiencias o historias que cuentan los testigos y actores directos 
Revista Herencia, Vol. 35 (1), enero-junio, 2022.

acerca del significado de los hechos vividos. Por esa razón, la Historia, antes de ser escrita, primero es narrada. El presente artículo pone estas tres formas en juego.

Por otra parte, el mismo Diccionario (2009), tiene la entrada de la categoría "memoria", escrita por Marcela Valdata, donde explica que: "Hablar de memoria implica remitir a un pasado que en algún momento, y por alguna situación determinada, quedó en el olvido. Un pasado que entra en acción necesita de alguna articulación para devenir en memoria" (173). Es en el campo de la historia como disciplina científica donde se realiza la re-construcción de las memorias como diferentes modos de representación de los acontecimientos del pasado.

Metodológicamente, este artículo recupera las memorias de los adultos mayores entrevistados por Ana Vera Estrada en el artículo "Historia contada de la vieja Habana o La Habana relatada" (2004) y contextualiza estos recuerdos con una mirada más compleja y enriquecida de esta ciudad que fue proclamada Patrimonio Cultural de la Humanidad por la Organización de las Naciones Unidas para la Educación, la Ciencia y la Cultura (UNESCO), el 14 de diciembre de 1982.

\section{¿Hasta dónde llega la ciudad?}

En el libro La Habana: magia de mi ciudad (1998) de Carmen R. Alfonso Hernández describe que:

[Se cree] que la villa de San Cristóbal de La Habana fue fundada por abril de 1514, a orillas del río Onicajinal (Mayabeque) en la costa sur del país, y en ese lugar, permaneció durante cinco años. Más tarde se trasladó al norte en dos asentamientos distintos, uno junto a la Chorrera -a la orilla derecha del río Casiguaguas (Almendares)-, y otro al lado del puerto, donde quedó definitivamente (1998, p. 5).

Por su ubicación, fue asediada continuamente, y se hizo necesario erigir fortalezas y levantar una muralla a su alrededor para su protección. Esta construcción duró dos siglos (el Real Castillo de la Fuerza 1558-1577; el Castillo de los Tres Reyes del Morro 15891610 y el de La Punta 1589-1630). Entre 1763-1774 se construyó el Castillo de San Carlos de la Cabaña, que era otra fortaleza de grandes proporciones (Alfonso, 1998, p. 6).

Hoy, para entender esta ciudad, menciona Ana Vera Estrada que:

La Habana Vieja se localiza en el extremo oriental de la península que cierra por el Oeste la entrada de la bahía. Varias calzadas de importancia, paralelas entre sí, y orientadas de Norte a Sur, delinean su entorno por la parte de tierra, como semicírculos cuyo vértice común se sitúa en el área del asentamiento más antiguo, aproximadamente localizable hoy entre las calles Empedrado y Luz, de Este a Oeste y Bernaza y la Avenida del Puerto, de Norte a Sur. Todas ellas tributan a la calle Monte, por donde se abrió la ruta principal hacia el sur de la provincia ya desde el siglo XVI (Estrada, 2004, p. 87).

Explica Carmen R. Alfonso Hernández que el primer asentamiento de población apareció en intramuros, en torno a la Plaza de la Ciénega, hoy de la Catedral, allá por las primeras décadas del siglo XVI. La zona extramuros fue necesaria para la expansión habitacional, la cual comenzó a urbanizarse hacia la segunda mitad del siglo XVIII, desbordando el límite amurallado. Todo lo demás era considerado "el campo". La ciudad se fue poblando y 
Revista Herencia, Vol. 35 (1), enero-junio, 2022.

la urbanización desarrollada en los extramuros hizo necesario el derribo de las murallas que dividían la ciudad en dos: La Habana Vieja y La Habana Moderna. El incremento de las viviendas y comercios continuó. Se estima que a principios del siglo XIX en intramuros habitaban unas cuarenta y cuatro mil personas, de las cuales veintiséis mil eran negros y mulatos y una población casi igual poblaba los grandes arrabales de Jesús María y la Salud (Alfonso, 1998, p.4).

Retoma Estrada este comentario de sus informantes: "La Habana Vieja se veía como un lugar muy pobre, con casas en muy mal estado y población hacinada, nadie iba allí a nada" (2004, p. 89). Sin embargo, otra informante se refiere a la existencia de dos partes o zonas claramente diferenciadas, tomando como eje la calle Muralla (famosa por sus grandes almacenes). Había una diferencia entre la arquitectura de los barrios del norte y los barrios del sur. El norte era residencial, con viviendas habitadas o construidas para una pequeña burguesía acomodada; era también zona de bancos, oficinas del Gobierno, bufetes de abogados y exhibe edificios magníficos.

El sur, sin embargo, era más insalubre y superpoblado, y albergaba a una variada población cubana y extranjera de escasos recursos, en contacto cotidiano con la prostitución y marginalidad o formando parte de ellas. En el barrio norte predominaban las viviendas individuales y en el sur, las colectivas; los tipos de vivienda se entremezclan en ambos lugares. Había diferentes clases de viviendas múltiples, solares, ciudadelas y casas de inquilinato (Estrada, 2004, p. 98). Esta división o estratificación de la ciudad muestra una característica que de Garay (2009) llama "copiosidad", es decir, la oralidad es redundante o copiosa, pues se basa en la repetición para preservar la continuidad o línea de pensamiento y así garantizar su comprensión. Los habitantes de La Habana no necesitaban de rótulos para poder diferenciar la estratificación La Habana Vieja.

Los contornos de la Plaza de Armas estaban conformados por un barrio tranquilo y silencioso. La Plaza de la Catedral tenía cerca pequeños comercios de víveres, la bodega, el puesto, la pescadería, etc. Estos eran sitios de encuentro entre los vecinos en su cotidianidad. Aquí estaba también el foco principal del turismo que llegaba a Cuba en la década de los cincuenta. El límite era la calle Muralla, donde residía gran cantidad de prostitutas. Todavía más hacia el sur, hacia extramuros, el barrio Jesús María era considerado una zona muy mala, pues eran los recintos principales de marginalidad.

Los informantes de Estrada hablaban de un ayer, a partir de su hoy. Pero, ¿cómo era esta ciudad que ellos retoman en sus memorias? Para explicarlo, Niklas Völkening, Andreas Benz and Matthias Schmidt, en el artículo "International Tourism and Urban Transformation In Old Havana" (2019), plantean una comparación sobre el "touristification" (la "turistificación" -una palabra que los autores acuñan-) de La Vieja Habana. El objetivo de su trabajo es analizar el proceso de transformación urbano espacioestructural, de manera que se pueda establecer las relaciones entre el turismo internacional como forma de entrada de divisas y el rol del gobierno de Cuba como facilitador de este proceso, así como de los programas de reconstrucción; para lograrlo, se basan en un trabajo anterior, realizado por Wehrhahn and Widderich en el año 2000 y publicado en la misma revista en alemán. Si bien este artículo es técnico (en términos de uso del espacio urbano), pues incluye mapas y explicaciones cuantitativas de uso espacial, cantidad de habitantes, 
Revista Herencia, Vol. 35 (1), enero-junio, 2022.

características físicas de las edificaciones y su relación con la parte económica, es pertinente rescatar de este artículo lo que sigue:

En las décadas siguientes a la Revolución Cubana en 1959, el mantenimiento -por no decir el desarrollo- de la Vieja Habana fue negligente, lo cual hoy paradójicamente, parece ser un aspecto seminal de su turismo potencial. El gobierno cubano se concentró en grandes proyectos habitacionales y facilidades industriales en las márgenes de La Habana (Foster, 2003, 788), lo cual, en combinación con la escasez de materiales constructivos, resultó en una decadencia estructural y un eventual colapso de las edificaciones en toda la Habana Vieja. En el 2008, cerca de un $60 \%$ de los edificios en esta área estaban en malas condiciones, y 420 edificios colapsaron durante ese año (Chinea et al, 2008, 12)1․ (Völkening et al., 2019, p. 89). La traducción es propia.

La importancia de la cita anterior radica en que describe la situación en la que se encontraba La Habana Vieja en el momento en que los entrevistados de Estrada cuentan y recurren a su memoria. Si bien, el espacio físico relatado es el mismo, de alguna manera ya no lo es.

Para tener una idea de la situación en la que se encontraban los informantes, Annyyret Nickel, en el artículo "El Casco Histórico de La Habana: La situación de vivienda y los conceptos de renovación urbana en La Habana" (1990) lo describe así:

De las 37 edificaciones de una manzana situado (SIC) en el suroeste del antiguo centro de la ciudad (ver cuadro 2), se encuentran cerca del 54 por ciento en un estado arquitectónico en muy malas condiciones; es decir más del 20 por ciento de las superficies ocupadas por techos, paredes y pisos en cada edificación, están deteriorados. En 17 edificaciones se construyeron azoteas para ampliar el espacio habitacional y en cada dos unidades de vivienda se colocaron entretechos. Un 23 por ciento de las 165 viviendas carece de instalaciones sanitarias y/o de cocina, es decir éstas se utilizan en forma colectiva y se encuentran por fuera de la unidad habitacional. Las instalaciones de infraestructura existentes, como desagües y red de teléfonos están sobrecargadas. Según el censo de 1981, 2.5 por ciento de las viviendas en La Habana no cuentan con instalaciones sanitarias y 35.5 por ciento son usadas en forma colectiva, como también un 24.7 por ciento [...]. La falta de espacio habitacional ha llevado a una construcción de los patios interiores y al desplazamiento de los empresarios de comercio al pormenor. Una parte de las construcciones libres por la demolición de la sustancia de construcción deteriorada, se usa inadecuadamente, así para talleres de reparación de autos y otros talleres que contaminan el medio ambiente [...]. La Habana Vieja con una densidad de población de casi 23,000 hab/km2 es el municipio más densamente poblado de la ciudad de La Habana, después de Centro Habana (Nickel, 1990, 81).

\section{¿Quiénes viven ahí? Demografía}

Dice Julio Le Riverend en el capítulo "Cuba: del semicolonialismo al socialismo (19331975)" que:

La información sobre el nivel de vida de la población antes de 1959 no abunda ni es de mucho fiar. Para simplificar, podríamos decir que las cifras aproximadas de muy diferentes fuentes, no se diferenciaban notablemente de las que han caracterizado los restantes países de América Latina, ni siquiera en lo aparente. En Cuba, como en otros países latinoamericanos, había una capital, con una fuerte proporción de la población total, con grandes construcciones turísticas y

\footnotetext{
${ }^{1}$ In the decades following the Cuban Revolution in 1959, the maintenance - not to speak of development - of Habana Vieja was neglected, which today turns out paradoxically to be a seminal aspect of its touristic potential. The Cuban government concentrated on large housing projects and industrial facilities on the fringes of Havana (Foster 2003, 788), which, in combination with a scarcity of construction materials, resulted in structural decay and eventually in frequent collapses of buildings all over Habana Vieja. In 2008, about $60 \%$ of the buildings in the area were in bad condition, and 420 buildings collapsed during that year (Chinea et al 2008, 12).
} 
Revista Herencia, Vol. 35 (1), enero-junio, 2022.

de la gente adinerada. Predominaban las escuelas privadas, por razón de clase y también por el grado inconcebible de deterioro y escasez de la escuela estatal. No había entonces un solo hospital rural y los de las capitales se hallaban escasos de capacidad y de recursos. Desde luego, la mortalidad infantil era muy alta. Mientras aumentaban los casinos y los prostíbulos (Le Riverend, 1984, p.55).

Hay que devolverse un poco más en el tiempo, y según explica Julio Le Riverend en el libro Historia económica de Cuba (1985), desde inicios del siglo XX había una gran inmigración de trabajadores contratados para ocuparlos en labores agrícolas, incluso se decretaron órdenes militares (como la del 15 de mayo de 1902) para prohibir la "importación" de haitianos, jamaiquinos y chinos. Según las cifras oficiales, entre 1913 y 1921, arribaron unas 150000 personas: la mitad haitianos y la otra mitad jamaiquinos. Después de la gran depresión (1929-1932) el Gobierno tomó la resolución de repatriar obligatoriamente a los antillanos residentes en el país (decreto de 19 de octubre de 1933); sin embargo, los datos y cifras oficiales no parecen exactos, pues da más la impresión de que empezaron a formar parte de la demografía nacional, con lo que se garantizaba la mano de obra necesaria para la industria (Le Riverend, 1985, p.563). Dice este autor: "En general, el peso del corte de las cañas lo llevaban sobre sus hombros los antillanos, mientras los inmigrantes blancos o nativos se concentraban en las explotaciones no azucareras o en la parte industrial de la producción de azúcar" (1985, p. 566). Luego, se empieza a dar una emigración rural hacia las ciudades, especialmente La Habana. De aquí que es posible pensar que se ubicaron justamente en esta área.

Y, hasta la fecha, este espacio físico, La Habana Vieja, sigue siendo un campo complejo para analizar en cuanto su demografía. Explica Carlos García Pleyan, en el texto "El mercado inmobiliario en cuba [SIC]: carencias legislativas y tributarias" (2020) lo siguiente:

\footnotetext{
Una de las zonas donde se evidencia un mayor impacto físico de las transformaciones es la de la Habana Vieja, donde ya se evidencian síntomas de gentrificación. Hay fuertes inversiones privadas en la rehabilitación de edificios viejos para destinarlos a alojamiento turístico o servicios al turismo (comercio, gastronomía) y la escala de las inversiones va creciendo económica y físicamente (en un inicio se compraban apartamentos o casas individuales mientras que en la actualidad se dan casos ya de compra de edificios enteros y partes de manzanas ...) (García, 2020, p.144).
}

Este autor considera que esto tiene sus luces y sus sombras. La ventaja es que mejora la apariencia de las edificaciones gracias a su rehabilitación. Sin embargo, también tiene la desventaja de que se da una creciente diferenciación social y la redistribución de la población en el territorio urbano en función de su capacidad económica. Justamente, de alguna manera, es el proceso inverso de lo que están relatando los entrevistados por Vera Estrada. La Habana Vieja era un espacio estratificado, pero tendía a una población de bajos ingresos económicos.

\section{El Comercio}

Explican los informantes de Ana Vera Estrada que, cerca de la calle Tejadillo, había muchos bufetes, lo cual hacía que fuera un lugar muy concurrido de día y muy tranquilo de noche. En este barrio existía "una gama amplia de comercios: había mucho comercio chino, mucho 
Revista Herencia, Vol. 35 (1), enero-junio, 2022.

comercio español ... almacenes, bodegas, que es lo más chiquito... casi todo era de españoles; de cubanos había muy poco. Los chinos tenían aquí muy buenos almacenes, en la calle de Aguacate y Lamparilla había uno muy grande, otro en Aguilar y Teniente Rey [...] Muchas carnicerías casi todas de españoles, [así como] los puestos de fruta eran chinos" (Estrada, 2004, p. 91).

También, en el barrio de Belén, al sur de la calle Muralla (entre la calle de Luz -donde estaba el convento de Belén- hasta San Isidro), se asentaban los comerciantes judíos. Se les llamaban "polacos": cualquiera que no fuera español, pero sí europeo, entraba en esa definición: rusos, franceses, alemanes, búlgaros, rumanos, etc. Dice de Garay (2009) "la oralidad resignifica en el presente real de su enunciación los sentidos del lenguaje" (198).

Sobre este tema, Julio Le Riverend en el libro mencionado anteriormente, aclara que la inmigración fue un factor decisivo en el desarrollo del proceso republicano de crecimiento de la población:

\begin{abstract}
El núcleo fundamental de estos inmigrantes es español, constituyendo un $62.7 \%$, siguiéndole la inmigración jamaiquina y haitiana. La inmigración norteamericana, aunque cuantitativamente importante, está compuesta de administradores, funcionarios y técnicos de las empresas establecidas en el país. La inmigración europea es típicamente urbana y comercial y excluyente, aun cuando no sea totalmente inasimilable. Similar a esta pero con más capacidad para asimilarse y dedicarse a ocupaciones no meramente comerciales, es la procedente de Siria y Líbano, que es uno de los grupos numerosos entre las inmigraciones nacionales (Le Riverend, 1985, p. 567).
\end{abstract}

Sin embargo, Estrada rescata de la memoria de los informantes que, entre bancos y comercios, el trabajo escaseaba. "La opulencia de los bancos y ministerios coexistía con una enconada lucha por la sobrevivencia. No porque no hubiera trabajo, sino porque las oportunidades se distribuían de modo desigual" (Estrada, 2004, p. 99). Los puestos de trabajo en ministerios y oficinas públicas, relativamente bien remunerados, no eran para todos los pobladores, aunque tuvieran alguna preparación, menos para los pobres, los negros y los sin oficio. Y había mucha corrupción en la asignación de puestos. Recuerdan los informantes que los negros tenían pocas posibilidades; por la razón del color de piel, por ejemplo, no podían ejercer como serenos, quienes eran todos españoles, y en cada cuadra había uno. Sin embargo, en el puerto la situación racial era distinta, pues allí sí tenían trabajo, como cargadores y estibadores.

Lo anterior es confirmado por Tomás Fernández Robaina en el libro El negro en Cuba 1902-1958 donde dice que "A pesar de estar la lucha social centralizada en las reivindicaciones obreras [...] la batalla contra la discriminación continuó desarrollándose" (Fernández, 1994, p. 150); a modo de ejemplo, este autor comenta que, a pesar de las denuncias contra los actos concretos de discriminación racial, estos continuaban; por ejemplo, un juez exoneró de toda responsabilidad a la dueña de una peluquería que no quiso ofrecer sus servicios a una ciudadana negra.

En el caso de las mujeres, las posibilidades eran pocas: algunas costureras, sombrereras, zapateras, y pocas eran oficinistas en el gobierno. Recuerda un informante, Roberto, que en algún momento se hizo una ley, durante el gobierno de Carlos Prío Socarrás, presidente de la República de 1948-1952, donde obligaron al comercio a contratar mujeres, pero, también menciona que solamente "en las tiendas metieron a unas cuantas negritas". Es importante aclarar que la época en estudio coincide con la II Guerra Mundial, la cual "desarticuló 
Revista Herencia, Vol. 35 (1), enero-junio, 2022.

inicialmente el sistema de comercio de Cuba. La súbita desaparición de nuestros mercados europeos, la falta de transporte y la escasez general de ciertas materias primas fueron factores inmediatos que comenzaron a pesar sobre la economía cubana desde 1940" (Le Riverend, 1985, p. 641). Por otra parte, el acceso a la educación era bastante limitado también; por ejemplo, cuenta Ismaela, una mulata de barrios del sur, que ella no pudo ir a la escuela, sino que aprendió a leer y escribir porque su esposo le enseñó.

La economía cubana estaba basada en la producción azucarera que se vio fuertemente sacudida por la situación mundial. "Los problemas planteados por la guerra respecto al abastecimiento -agravados por un alza súbita del consumo- fueron incentivo suficiente para el desarrollo de nuevas actividades económicas y agrícolas" (Le Riverend, 1985, p. 644). Menciona el mismo autor que durante la II Guerra Mundial, Cuba debió buscar nuevos quehaceres y formas de autoabastecimiento, luego de la guerra: "En lo sustancial, la estructura permanecía intocada, y, aún más, al amparo de las condiciones de guerra y de posguerra, se fortaleció un nuevo latifundio -el arrocero-, se expandió el latifundio ganadero y el comercio quedó como siempre concentrado en la exportación de azúcar" (Le Riverend, 1984, p. 52).

Sin embargo, esta situación cambia radicalmente con la Revolución. Francisco López Segrera (1989) en el libro Cuba: Cultura y sociedad 1510-1985 describe puntualmente la situación económica de Cuba antes de enero de 1959:

El comercio exterior estaba contralado por Estados Unidos -que proveía el 75\% de nuestras importaciones y adquiría el $66 \%$ de las exportaciones cubanas-: los principales renglones de nuestra economía $-50 \%$ de la producción azucarera, minas, banca, tierra, etc.- estaban casi totalmente en manos norteamericanas [...] la economía estaba subordinada al azúcar, que representaba cerca del $80 \%$ de las exportaciones y del 30 al $40 \%$ de la renta nacional; y había un promedio anual de 549000 desocupados [Julián Alienes en su Tesis sobre el desarrollo económico considera que son 700000 personas] de una fuerza de trabajo de 2204000 (López, 1989, p. 198).

\section{Tres hitos puntuales: diamantes, National City Bank y la llegada de la revolución}

Para entender la Historia (con mayúscula) cubana, es necesario pasar por los recuerdos de quienes vivenciaron los acontecimientos, hitos y hechos que cambiaron el rumbo de esta nación. De Garay (2009), cuando habla de la oralidad dice que:

la comunicación oral depende de la memoria para su reproducción. Pero esta aparente desventaja, no debe interpretarse como una debilidad o pérdida de la identidad de la memoria. Por el contrario, la memoria debe comprenderse como un proceso de constante reformulación y actualización de sentidos (2009, p. 199).

Los comentarios de los informantes son vitales, pues vivieron, como testigos oculares, los acontecimientos relatados. Por las circunstancias políticas cubanas, durante mucho tiempo este recuerdo no fue posible decirlo en voz alta sin correr riesgos graves; sin embargo, gracias a la entrevista de Estrada, el recuerdo abre la posibilidad para la investigación de los tres hitos seleccionados de la Historia. 
Revista Herencia, Vol. 35 (1), enero-junio, 2022.

Uno de los temas mencionados por los informantes, y es muy llamativo, es el tráfico de diamantes. Ana Vera Estrada aclara que ella "no había oído nada del tema, pero que es algo que la ciencia social puede retomar y convertir en objeto de indagación" (Estrada, 2004, p. 92). Sin embargo, otros textos sí mencionan el tema de los diamantes; por ejemplo, Julio Le Riverend en el libro Historia Económica de Cuba menciona que:

muy ilustrativo del tipo de industrias resultantes de la situación internacional es el caso de la talla de los diamantes que tuvo en La Habana un centro de refugio muy importante, desapareciendo casi por completo en cuanto la cesación de la guerra permitió restaurarla en los países europeos que han constituido tradicionalmente su centro (Le Riverend, 1985, p.644).

Esto lo confirma también Theo Scharf en el artículo "L'industrie du diamant a Cuba" explica que la Segunda Guerra Mundial causó numerosos desplazamientos de las industrias en el mundo, de las cuales una de ellas fue la de la talla de diamantes. Los grandes productores holandeses y belgas querían irse antes de que los alemanes tomaran sus materiales para su uso en la Guerra. En esta corriente de refugiados (muchos de ellos habían pertenecido a esta industria), buscaban migrar hacia Nueva York, pero debido a las restricciones migratorias, se vieron obligados a quedarse en los puertos de escala, como Brasil o Venezuela; "pero el centro más importante fue La Habana, más cerca de Nueva York, la cual se convirtió en una bolsa de diamantes en tiempos de guerra, ahí se encontraba un número elevado de obreros especializados y la mano de obra local era barata" (Sharf, p. $63)^{2}$ (la traducción es propia). La maquinaria que se usaba fue copiada de las belgas y neoyorquinas, y ajustada a las necesidades; los talleres nacionales proveyeron a las fábricas de todos los accesorios. Sin embargo, ya hacia el año de 1946-1947 se da el retorno a Bélgica.

Por otra parte, uno de los informantes de Ana Vera Estrada describe haber presenciado personalmente el robo del Tesoro de la República, el 28 de diciembre de 1958. Según este señor, el National City Bank estaba entre O’Reilly y Compostela, [según la historia del banco, la sucursal quedaba en la Calle Obispo y Obrapía]. Faltando 10 minutos para las 3 el banco cerraba a las 3- llegaron unos hombres con maletas, apoderados de Batista, y sacaron de las cajitas de seguridad 20 maletas, en las que llevaban 375 millones [no dice la moneda] y quedaron 125 millones de los 500, que fue lo que recibió Felipe Pazos y duró hasta que llegó el Che ${ }^{3}$. Esto puede ser posible, pues Julio Le Riverend menciona que "El dictador [refiéndose a Batista] huyó con sus principales cómplices en la madrugada del 1 de enero de 1959 cuando ya no quedaba duda de que Santiago de Cuba y Santa Clara caían en manos de los rebeldes" (Le Riverend, 1984, p. 57); sin embargo, no hace la relación con el relato del informante y en ninguno de los otros textos revisados se menciona este robo.

Sobre el tercer hito, la llegada de la Revolución a la ciudad, Julio Le Riverend en el capítulo "Cuba: del semicolonialismo al socialismo 1933-1975" publicado en el libro América

\footnotetext{
${ }^{2}$ Mais, le centre le plus importante devint La Havane, plus proche de New York, devenue borse du diamant en temps de guerre; il s'y trouva un nombre élevé d'ouvriers spécialisés; el la main-d'œuvre locale était à bon marché (Sharf, p. 63).

3 Según Carmen R. Alfonso Hernández (1998) en el libro La Habana: magia de mi ciudad, el comandante Guevara, conocido como el Che Guevara, al frente de la columna 8 del Ejército Rebelde llegó a la capital. Desde finales de noviembre de 1959 hasta el 23 de febrero de 1961 ocupó la presidencia del Banco Nacional de Cuba, situado en la calle Cuba, en La Habana Vieja.
} 
Revista Herencia, Vol. 35 (1), enero-junio, 2022.

Latina: Historia de medio siglo 2: Centroamérica, México y el Caribe (coordinado por Pablo González Casanova) explica que al recapitular lo sucedido en Cuba, desde 1902 se observa una suerte de diástole y sístole política, en la que los gobiernos conservadores y liberales alternaban, de modo que cada cual llamaba a una mentida reparación de las fechorías del otro. En 1940, se inicia el proceso de llamada a una Asamblea Constituyente, y el presidente para la época era Fulgencio Batista y Zaldívar (1940-1944). Le seguirían Ramón Grau San Martín (1944-1948), luego Carlos Prío Socarrás (1948-1952) y, nuevamente, Batista pero por golpe de Estado, de 1952-1959, hasta la llegada de Fidel Castro, el 1 de enero de 1959. "El período comprendido de enero de 1959 a octubre de 1960 revela los contenidos de una revolución agraria y antiimperialista, a lo cual se le consuman algunas de las significativas medidas democrático-burguesas" (Le Riverend, 1984, p. 58). Después, continuaría la nacionalización total de la economía, de la propiedad inmobiliaria urbana y de la enseñanza.

Justamente, en esto coincide, Luis E. Aguilar en el capítulo “Cuba, c. 1860-1934” del libro de Leslie Bethel, Historia de América Latina, tomo 9: México, América Central y El Caribe 1870-1930, donde explica la situación política prerrevolucionaria en los siguientes términos:

\begin{abstract}
Con todo, la agitación del período 1930-1934 resultó ser mucho más que otro episodio de violencia política en Cuba. Las fuerzas nacionalistas, sociales y políticas desatadas durante el conflicto transformaron la isla y dieron comienzo a una nueva era. Los líderes, los partidos y las ideas que aparecieron en 1933 dominarían y controlarían los destinos de Cuba durante los siguientes 25 años. La sociedad cubana que Fidel Castro encontró en 1959 e incluso la subida al poder del propio Castro no pueden entenderse sin tener en cuenta los profundos efectos que la frustrada revolución de 1933 surtió en la historia de Cuba (1992, p. 239).
\end{abstract}

La Revolución cubana en 1959 abrió una nueva página en la historia de Cuba y también de América Latina. Se han escrito muchos libros que relatan el antes, durante y después de esta revolución, y ese no es el objetivo de este artículo. Sin embargo, llama la atención el silencio profundo de los informantes sobre este tema.

\title{
Última consideración
}

Mirar la historia de Cuba desde la distancia, en tiempo y en espacio, es difícil: lo es tanto antes como después de la Revolución Cubana. Ronald H. Chilcote resume esta complejidad de la siguiente manera:

La atención de los investigadores interesados en la Revolución Cubana de 1959 y en los años que siguieron a ella, es probable que se encuentre en temas como: 1) El papel que Estados Unidos jugó en la intervención, y el "imperialismo" tal como se refleja en los escritos de norteamericanos y latinoamericanos; 2) las rígidas estructuras que estancaron la economía con anterioridad a 1959 y, en particular, la relación comercial con los Estados Unidos y otros mercados extranjeros en la producción de azúcar cubano; 3) los bajos niveles de vida causados por la rígida estructura de clases, la mala distribución de las rentas y los problemas persistentes en educación, salubridad y habitación; estas condiciones han sido alteradas hasta cierto punto por la Revolución, y a grupos étnicos como el de los negros les ha sido permitido participar en el proceso revolucionario; 4) la Iglesia Católica, atrasada y conservadora, que antes de 1959 influyera en los sectores de la clase media urbana, ha establecido desde entonces un modus vivendi con el gobierno de Castro, pero continúa debilitada por la deportación de los sacerdotes españoles (aproximadamente dos terceras partes de todos los sacerdotes en Cuba); 5) su historia política, que ha sido señalada por políticos pragmáticos y partidos políticos que 
Revista Herencia, Vol. 35 (1), enero-junio, 2022.

abogaron más de una vez por el cambio estructural pero hicieron poco por realizarlo (1967, p.107).

Por esta misma razón, y debido a esta complejidad, son muy valiosas las entrevistas y la recopilación, aunque sean a partir de la memoria, que ha hecho Ana Vera Estrada en este texto, pues abre puertas para el estudio de una historia antes de la Revolución, se piense esta como triunfo o traición, pues depende de quién cuente la Historia, así se tomará una distinta posición, lo cual hace que sea realmente difícil de contar.

Por otra parte, también ese espacio llamado La Habana Vieja es un lugar complejo de la memoria, pues los informantes cuentan ese espacio físico de La Habana Vieja prerrevolucionaria desde el mismo espacio, pero sumamente deteriorado, por lo cual ya es otro totalmente distinto.

\section{BIBLIOGRAFÍA}

Alfonso Hernández, C. (1998). La Habana: magia de mi ciudad. España: Pablo de la Torriente Editorial.

Aguilar, L.E. (1992). “Cuba, c. 1860-1934” en Bethell, Leslie (ed.) (1992) Historia de América Latina, tomo 9: México, América Central y El Caribe, c. 18701930. Barcelona: Editorial Crítica.

Chilcote, R. (1967). "Cambio estructural y desarrollo en Cuba" en Instituto de Desarrollo Económico y social. Vol.7, No. 26 (jul-sep., 1967) pp. 107-147. https://www.jstor.org/stable/3465763

De Garay, G. (2009). “Oralidad” en Diccionario de Estudios Culturales Latinoamericanos. Mónica Szurmuk y Robert McKee (coord.) México: Siglo XXI Editores: Instituto Mora.

Estrada, A.V. (2004). "Historia contada de la vieja Habana o La Habana relatada" en Estrada, A.V. (comp.) (2004) La oralidad: ¿ciencia o sabiduría popular? La Habana: Centro de Investigación y Desarrollo de la Cultura Cubana Juan Marinello.

Fernández Robaina, T. (1994). El negro en Cuba: 1902-1945. Apuntes para la historia de la discriminación racial. Cuba: Editorial de Ciencias Sociales, La Habana.

García Pleyán, C. (2020). "El mercado inmobiliario en cuba: carencias legislativas y tributarias" en International Journal of Cuban Studies , Vol. 12, No. 1, Cuban Scholars on the Cuban Economy and Society (Summer 2020), pp. 135-148. https://www.jstor.org/stable/10.13169/intejcubastud.12.1.0135

Guerra, S. y Maldonado, A. (2009). Historia de la Revolución Cubana. Navarra: Editorial Txalaparta. 
Revista Herencia, Vol. 35 (1), enero-junio, 2022.

Le Riverend, J. (1984). “Cuba: del semicolonialismo al socialismo (1933-1975) en González Casanova, Pablo (coord.) (1984). América Latina: Historia de medio siglo 2: Centroamérica, México y el Caribe. Segunda edición. México: Siglo XXI editores S.A.

Le Riverend, J. (1985). Historia Económica de Cuba. La Habana: Editorial de Ciencias Sociales.

López Segrera, F. (1989). Cuba: Cultura y sociedad (1510-1985). La Habana: Editorial Letras Cubanas.

Nickel, A. (1990). "El Casco Histórico de La Habana: La situación de vivienda y los conceptos de renovación urbana en La Habana" en Revista Geográfica, juliodiciembre 1990, No. 112, pp. 75-90 https://www.jstor.org/stable/40992617.

Opatrný, J. (editor). (1995). Cuba: Algunos problemas de su historia. Praga: Centro de Estudios Ibero-Americano de la Universidad Carolina.

Pierre-Charles, G. (1978). Génesis de la Revolución Cubana. Segunda edición. México: Siglo XXI Editores, S.A.

Sharf, T. (s.f.). "L'industrie du diamant a Cuba" en Annales de Géographie, 58e Année, No. $309 \quad$ (Janvier-Mars 1949), pp. 62-64. https://www.jstor.org/stable/23441445 R

UNESCO. (1982). Report of the rapporteur. World Heritage Committee, Sixth Session. Paris.

Völkening, N.; Benz A.y Schmidt M. (2019). "International Tourism and Urban Transformation In Old Havana" en Erdkunde, April - June 2019, Bd. 73, H. 2 (April - June 2019), $\quad$ pp. 83-96. https://www.jstor.org/stable/10.2307/26663993 
Revista Herencia, Vol. 35 (1), enero-junio, 2022. 